\title{
Biodiversity and intraspecific genetic variation
}

\author{
Claes Ramel \\ Wallenberg Laboratory, Stockholm University, S-106 91 Stockholm, Sweden
}

\begin{abstract}
Biodiversity is mostly discussed at the level of species, but genetic variation within species may be as important as variation between species. It enables organisms to adapt to environmental changes. The requirement for genetic variation differs however widely between species and groups of species. Animal species at the top of the food web, such as large carnivorous mammals, tend to have a remarkably small genetic variation, presumably due to a constant ecological niche. Many other organisms must be genetically 'prepared' to meet environmental changes. As an example, for organisms relying on camouflage a colour polymorphism matching altered environments in space and time is of selective importance. Several mechanisms are involved in maintaining a genetic variation. One such mechanism is balanced polymorphism based on a higher viability of heterozygotes as compared to both homozygotes. A major part of DNA in multicellular organisms does not have any discernible function, but behave as parasites. Important elements in that respect are mobile DNA elements, 'jumping genes', which can move from one place in the genome to another, causing mutations at site of insertion and increased genetic variation. Some mobile elements have been shown to move from one individual and species to another asexually. The mechanism behind this 'horizontal transfer' is unknown, but presumably is mediated by microorganisms. Protective devices against these parasitic DNA elements have been developed by many organisms.
\end{abstract}

\section{INTRODUCTION}

Biodiversity is mostly discussed at the level of species and higher taxonomic categories. However, the foundation for biodiversity and organic evolution, is the genetic variation within species. This intraspecific variation provides the material for long term evolutionary adaptation and short term adaptation to seasonal and other rapid fluctuations in environmental factors. Human interference with the ecosystems has resulted in extensive alterations and fragmentations of habitats. Diminishing populations gives rise to inbreeding, genetic drift and a loss of genetic variation, which make the organisms vulnerable to environmental changes. Myer (1) has recently emphasised that loss of populations may be more fatal than loss of species. Hughes and Ehrlich (2) have estimated that 1800 populations get extinct per hour in tropical forests. In this paper I will discuss some aspects of intraspecific genetic variation; how organisms and populations loose, maintain or increase the genetic variation.

\section{NATURE OF GENETIC VARIATION}

During recent time our concept of DNA in higher organisms has been altered in two respects - namely the organisation of DNA and the stability of DNA. Concerning the organisation of DNA, the genome was previously visualised as a string of genes like pearls in a necklace, each one coding for a protein. However, it is now clear that only about three percent of DNA of man is made up of ordinary coding genes, and similar proportion of genes is prevailing in other vertebrates, as well as many invertebrates and plants. Some $45 \%$ of non coding DNA is indirectly involved in the coding and expression of genes, but for the rest, constituting over half of the genome, no clear function can be attributed. This lack of obvious function is reflected by the designation of this DNA as 'selfish', 'parasite', 'junk' and 'extra' DNA.

The ordinary coding genes are of course of particular importance in the present context as they are responsible for phenotypic expression of DNA. However lately more and more attention has been paid to the non coding or non-functional DNA. It was suggested by Orgel and Crick (3) that this DNA behaves as a parasite and it remains in the cell as long as it does not cause an excessive selective disadvantage. This DNA is of interest in the present context when discussing the occurrence and role of intraspecific variation

* Lecture presented at the International Conference on Biodiversity and Bioresources, Phuket, Thailand, 23-27 November 1997. Other presentations are published in this issue, pp. 2065-2145. 
for two reasons. First it comprises mobile genetic elements - 'jumping genes' or transposons, which have the ability to move from one place in the genome to another, causing mutations where they settle down and providing new genetic variation. Secondly this DNA to a large extent comprises repeated sequences of DNA, often exhibiting an extreme instability with mutation rates thousand of times higher than for ordinary genes. This repeated DNA is usually classified as microsatellites with repeat units below 5 and as minisatellites with repeat units of usually 9 to about 50 units. The high mutation rate of this satellite DNA has resulted in an extensive variation with each individuals having its unique pattern - 'fingerprinting'. The mini- and microsatellites constitute a powerful tool in population ecology and genetics to study issues like:

- Genetic relationship between individuals and populations;

- Reproductive strategies;

- Effective breeding populations

- Historic development of species and populations;

- Prerequisite for genetic drift and loss of genetic variation.

These issues are of obvious scientific interest but also of great importance for conservation biology.

The use of minisatellite fingerprinting technology in conservation biology can be examplified by an investigation of Waldrapp Ibis (Geronticus eremita) by Jeffreys and his group in Leicester in England (4). This ibis is one of the most threatened species of birds with only a few hundred specimens left. The survival of the species will probably be dependent on breeding in captivity. There are facilities with captive birds from incompletely known founders. As the genetic base of these captive birds probably is very narrow, it is essential to avoid inbreeding. With the help of fingerprinting of 39 birds the relationship between the birds could be established and in the further breeding process suitable and non related birds could be used, thus avoiding a decrease of viability through loss of genetic variation.

\section{GENETIC VARIATION IN DIFFERENT ORGANISMS}

The susceptibility to inbreeding and a loss of genetic variation varies greatly between different organisms. O'Brian reported 1985 (5) that the cheetah in Africa had a remarkable lack of genetic variation, measured as diversity of proteins, isozymes. Skin drafting between specimens did not elicit tissue rejection, indicating a lack of polymorphism of the immune system. It was also reported that the cheetah exhibits a low reproduction and other signs of inbreeding depression. The lack of genetic variation in this species could be explained by the cheetah having gone through a bottle neck in population size, which would have resulted in a loss of heterozygosity through genetic drift. However, the low reproduction as a sign of inbreeding has been questioned (6), claiming that the cheetah does not exhibit a low reproduction potential. The low net reproduction rather has other causes, particularly predation from lions and hyenas.

The low genetic variation of cheetah has turned out not to be specific for this species, but a general trait of large mammalian carnivors. Thus Larsen et al. (7) reported a similar low variability of proteins in polar bears. In later years a series of other mammalian carnivors has shown an even lower variability of proteins than the cheetah (6).

The extent of polymorphism and heterozygosity varies greatly between different groups of organisms. The large variance within the different taxonomic units indicates biologically and ecologically based divergence in the susceptibility to inbreeding and loss of genetic variation. The average level of genetic variation is low in mammals, and this low variation is particularly pronounced in large mammals.

\section{DOMINANCE AND OVERDOMINANCE}

The genetic variation in and between populations is the outcome of several factors, such as mutation rate, breeding size of the population, breeding strategy, migration and, above all, selection. The genetic diversity between individuals in a population is designated as polymorphism, and the variation within the individuals as heterozygosity. At the level of the gene, the occurrence of a genetic variation is dependent on the property of the alleles - whether they are dominant or recessive. A dominant detrimental allele will be eliminated through selection. The selection against recessive detrimental alleles without any dominant effect is slow and such alleles remain for long times in the population. The size of the population is critical 
for the maintenance of polymorphism of recessive and neutral alleles. Small populations are subjected to genetic drift that is, a random loss of alleles, which will affect the persistence of polymorphism and heterozygosity.

An important case, which operates towards preserving polymorphism and heterozygosity, is the occurrence of a higher viability of the heterozygote - named heterosis, hybrid vigour and overdominance, that is, the viability is: $+/+<+/ d>d / d$, where + is the wild type allele and $d$ recessive detrimental mutation. The homozygote $d / d$ can even be lethal. The fact that the heterozygote has a higher viability than both homozygotes will result in a balanced frequency of both alleles. The occurrence and interpretation of heterosis has been subjected to much discussions. This kind of balanced genetic variation nevertheless plays an important role both in natural populations and in domesticated organisms. A classical example in human populations is the occurrence of the allele for sickle cell anemia in Africa. In spite of the fact that the homozygotes for this allele suffer from severe anemia the allele remains in the population because the heterozygotes are resistant to malaria.

An old and well known practical application of hybrid vigour is the development of 'hybrid corn'. Different lines of corn are subjected to inbreeding. When these lines subsequently are crossed to each other, a highly heterozygote offspring is produced, which exhibits hybrid vigour and a dramatically increased yield. There are two interpretations of this process. During the inbreeding many detrimental genes are eliminated and remaining recessive detrimental genes will be 'covered' after the cross between the inbred lines. The second explanation is based on 'overdominance' that is, a higher viability by heterozygotes themselves.

Although there is no general explanation of the molecular background of overdominance, the occurrence of overdominance has been indicated experimentally in different ways. As one example I may bring up some radiation experiments that we made at our laboratory $(8,9)$. Inbred lines of fruitflies, Drosophila, were irradiated each generation with $3000 \mathrm{R}$. The viability was measured by counting the number of hatched larvae and the time for their hatching. In the beginning the viability of the irradiated populations decreased drastically, but gradually they recovered and after several generations of irradiation the viability had increased above the viability before the start of the experiment. The development of the viability of the irradiated population can be explained by induced mutations which increased the heterozygosity, causing heterosis. The successive increase of the heterozygosity from the radiation gradually overcame the dominant detrimentals that also were produced and which set down the net viability in the first generations. Experimental evidens of radiation induced overdominance was also obtained on inbred mice with irradiation of males for successive generations with 50 and $100 \mathrm{R}(10)$. As the indication of viability the gain in weight of the offspring was measured. After some generations the irradiated populations showed a significantly more rapid gain of weight of the offspring as compared to the unirradiated control, indicating a similar induction of heterosis.

\section{ADAPTIVE POLYMORPHISM}

Thus some organisms such as large mammals and mammalian carnivors are able to maintain a low genetic variability, presumably due to a sufficiently constant environment and feeding ecology, which does not require rapid adaptation to environmental changes. In contrast, for many other organisms a genetic polymorphism and a rapid alteration of phenotypic traits is necessary for their survival. There are several examples of such genetic changes. A classical case is the idustrial melanism among insects. Due to the development of polluting industries in the cities during the 19th century, dark colour among insects became of selective value because of the sooty background (11). The first black specimen of the normally light coloured moth Biston betularia was found in Manchester 1948 and already by 189598 per cent of the population in Manchester was black. Experiments with the two colour morphs has shown that birds primarily caught the light morph against the sooty background and the black morph against a background free from soot (12). There are also indications that the dark form of the moth preferably settle down against a black background (12). Several species of moths and also other insects showed a similar development of black morphs in industrial areas. With modern purification of industrial emissions, the air pollution with soot has diminished. Melanistic forms are consequenty getting more scarce.

There are many other examples of genetic alterations of natural populations as a response to human activities. A vital problem in that context is the generation of pesticide resistance, with severe 
consequences particularly in tropical regions. The emission of heavy metals selectively favours organisms which are able to build up a genetic resistance against the metals and this in its turn may alter the species composition in ecosystem polluted with heavy metals. Selection experiments with methyl mercury on Drosophila revealed a rapid response, giving rise to a very resistance strain after a few generations (13). Another example of human induced selection concerns the fritillary butterfly Ephydryas editha, which changed its genetically determined host plant because of human cultivation (14).

Many organisms are dependent on a genetic polymorphism in coloration for their protection against predators, such as mimicry and camouflage. The evolution of a camouflage pattern can be complex and favour an intricate polymorphism. For instance a uniform camouflage pattern is often inefficient as a protection if the prey occurs in a dense population - a specialist predator will learn how to localise the prey anyway (15). From the point of view of the prey there are two possibilities to overcome this problem either by restricting population density or by developing a polymorphic camouflage pattern, preventing the predator to develop a search image. Often such a polymorphism is also directed towards local and temporal variations in the background coloration. A well studied example of a balanced polymorphism of that kind is provided by the snails Cepea nemoralis and Cepea hortensis. The genetics of the colour and pattern of these snails have been worked out (16). The occurrence of the different colour morphs is at least partly due to genetic adaptation to the background vegetation and the selection by predators. Brown snails without banding pattern are predominant in wooden areas with a cover of brown leaves, yellow banded forms occur in pasture areas and shrubs etc.(17). A main predator on these snails in England is the song-thrush, Turdus philomelos. It has the habit of opening the snails at specific spots and the composition of the snails predated by thrushes could be compared with the actual snail population. There were significant differences between the two samples, indicating a selective, non random catch by the birds. However, the camouflage pattern and the selection by the predator does not seem to be sufficient to explain the balanced polymorphism in the Cepea snails. Another probable component is a higher viability of the heterozygotes (18).

Another example of a balanced polymorphism is the grasshopper Myrmeleotettix maculatus. This is a common species in Europe and it exhibits a pronounced colour polymorphism. It often occurs in dense populations and it can á priori be suspected that the extensive polymorphism is an adaptation to avoid the development of a 'search image' by the predators, primarily starlings, shrikes and other insectivorous birds (19). In a region studied in Sweden this grasshopper occurs in different habitats, from luxuriant pastures to dry and brown step, grazed by sheep. By a simplified classification in green and non green grasshoppers it was found that the frequency of green grasshoppers varied from close to 80 per cent in the green pastures to about 10 per cent on step areas grazed by sheep, where the vegetation rapidly turns brown during the summer. On the step area, significanly fewer green grasshoppers were recorded where sheep had been grazing as compared to a non grazed area just on the other side of a fence. Significantly higher frequency of red coloured grasshoppers was observed in some areas, presumably as an adaptation towards the background vegetation with abundant occurrence of Thymus serpyllum with red flowers.

\section{LOSS, MAINTENANCE AND INCREASE OF GENETIC VARIATION}

Deprivation of a genetic diversity in a population can occur by an altered selection against some genotypes and by random loss through inbreeding in small populations. An occasional population 'bottleneck', resulting in genetic drift, can be traced for a long space of time, particularly in species with a low reproductive capacity, as indicated by the cheetah. Most crossbreeding species are adapted to a high frequency of heterozygosity and a loss of heterozygosity by a diminished population size and genetic drift often has a negative effect on the viability of the whole population. This is a problem of great current importance, when so many habitats are fragmentated by human activities. The relation between population size, loss of genetic diversity and viability can be illustrated by experiments on Drosophila (20). In an experimental population of Drosophila $74 \%$ of reproductive fitness was lost after 11 generations. Introduction of one single fly from another unrelated population increased the fitness from 0.29 to 0.63 . In another experiment a population of 1000 flies lost $85 \%$ of the genetic variation in 2.5 years and the effective population consequently became considerably smaller. Recent observations indicate that loss of genetic variation, leading to extinction of sensitive species by environmental stress situations can occur, even if the population size is above the conventional limit for genetic drift. 
How do organisms maintain heterozygosity and compensate for loss of genetic variability? Important mechanisms are higher viability of heterozygotes than both homozygotes and different kinds of balanced polymorphism as touched upon above.

But new genetic variation is provided by immigration from other populations (metapopulation) and by mutations. Beside 'conventional' mutations like point mutations, frame shifts, deletions, translocations and other chromosomal aberrations, the new insight in the organisation and behaviour of DNA at large has revealed a far more dynamic property of DNA than was realised previously. Many of these alterations occur at frequencies far above conventional mutations. Amplifications of DNA is such an alteration, which has attracted a great deal of attention during the last few years. At the level of coding genes amplification is one method with which the organism may counteract an environmental stress. Exposure to heavy metals can elicit an amplification of metallothionein genes, which produce a protein which binds to heavy metals like mercury and cadmium. In Drosophila it was found that amplification of the genes for superoxide dismutase and catalase, which protect against free radicals, increased the viability; the flies became more fit, they lived longer, moved faster at high age and had an increased metabolism (21).

As mentioned before, over 50 per cent of the genome of mammals and many other organisms have no obvious biological function and is mostly organised as repeated sequences of DNA. The parasitic nature of that DNA is indicated by the fact that some organisms have developed specific protective devices against this DNA. Thus fungi like bread mould, Neurospora, get rid of repeated DNA sequences by causing mutations through methylation of cytosin ('RIP - Repeat-induced Point Mutations) (22). Also in mammals a 'silencing' of mobile elements occur by means of methylation. A demethylation of mobile elements occurs in tumour dells, giving rise to a genetic instability and a high frequency of mutations (23)

An element of this 'extra' DNA, which is of particular interest in the present context is transposing elements, that is mobile DNA or 'jumping genes'. These jumping genes were first recognized and described by Barbara McClintock fifty years ago, for which discovery she received the Nobel Prize 1983. McClintock made her observations on maize, but in the 1970s it was clear that transposing elements presumably occur in all organisms. In humans there are hundreds of thousand potentially mobile DNA elements. The mobile elements can move from one place to another in the cell, causing mutations where they get inserted. Two major groups of transposing elements can be recognized -those that move as DNA from one place to another, and those that are organised as retroviruses, moving as RNA and getting inserted after conversion from RNA to DNA through reverse transcriptase. In some organisms transposing elements play a dominating role for spontaneous mutations. In Drosophila over 50 per cent of spontaneous mutations are due to these mobile elements. In one case that we have studied (24) a part of the $\mathrm{X}$ chromosome in Drosophila, covering two genes, had become mobile and insertions to over 250 places in all chromosomes could be recorded. Of interest is the observation that a neighbouring gene could be included in the movement- a phenomenon named 'hitchhiking'.

Also mobile DNA elements can be considered parasites in the cells and protection against them have been developed. Thus they are inactivated by methylation of DNA in somatic cells. It is of interest that a demethylation occurs in tumour cells, which is one reason for the extreme genetic instability and mutation rate in tumour cells. Some organisms seem to have a high resistance to mobile elements. Thus in maize over 50 per cent of DNA is constituted by retrovirus-like elements, which have been silenced and do not move.

However, activation of transposing elements may sometimes have a selective function. Different kind of stresses tend to induce transpositions, and interestingly, inbreeding can have this consequence (25). In an experiment by Biémond et al (26) the movement of the mobile element copia in Drosophila was studied in highly inbred populations for 70 generations. At one point there was a burst of movements of copia. It was interpreted as an adaptation to counteract the inbreeding by inducing insertion mutations and heterozygosity.

When dealing with genetic variation within species it may be of special significance that transposing elements do not only move within the cells, but there are many indications for horizontal and non sexual transfer of these elements between species. Sequencing of DNA of such elements has shown closer similarities between widely different organisms than between closely related species, strongly pointing to a horizontal transfer. This can for instance be concluded from the observation that insects and plants that they live on share the same transposing elements. The transposing element P-factor in Drosophila has 
spread to virtually all natural populations since the beginning of the century and this has also involved a transfer between different Drosophila species. In one case a transfer of the P-factor by means of mites between Drosophila was observed (27). A particularly illustrative example of horizontal transfer is provided by the mobile element mariner (28), which was first recorded in the Drosophila species Drosophila mauritiana. It turned out that this element existed in many insects and other arthropodes. The DNA sequence of the element showed a close correlation with the evolutionary relationship between the species and an evolutionary tree could be constructed on the bases of DNA. It could be concluded that mariner must have been formed about 250 million years ago. This tree based on mariner was in accordance with an evolutionary tree based on conventional systematic data, except on one point - bees and earwigs had almost identical mariner in spite of the fact that they separated 150 million years ago. The element must have been transferred from one species to the other in recent time. Other deviations from expectation were found, indicating a horizontal transfer. Recently mariner has also been found in humans (29) and also there horizontal transfer between individuals is indicated.

The actual mechanism behind this horizontal transfer of DNA is not known, but it can be assumed that microorganisms, bacteria and/or virus are involved. In conclusion it can be stated that transposing elements contribute to an increase of genetic variation within species, but through horizontal transfer also between species. This horizontal transfer between species violates the current notion that established species with separate gene pools never exchange genetic information with each other. How important this mechanism of increasing genetic variation by means of mobile DNA elements has been during the course of evolution, nobody knows.

\section{REFERENCES}

1 N. Myer. Science 278, 597-598 (1997)

2 Hughes, G.C. Daily and P. Ehrlich. Science 278, 689-692 (1997).

3 L. E. Orgel and F.H.C. Crick. Nature 284, 604-607 (1980).

4 E.N. Singer, C.R. Schmidt and A.J. Jeffreys. Molecular Ecology 3, 291-300 (1994).

5 S.J. O'Brian, D.E. Wildt, D. Goldman, D.R. Merril and M. Bush. Science 221, 459-462 (1983).

6 M. Merola. Conservation Biology 8, 961-971 (1994).

7 T. Larsen, H. Tegelström, R. Kumar Juneja and M.K. Taylor. Polar Research, 97-105 (1983).

8 G. Bonnier, U.-B. Jonsson and C. Ramel. Hereditas 44, 378-406 (1958).

9 G. Bonnier, U.B. Jonsson and C. Ramel. Hereditas 45, 441-448 (1959).

10 C. Ramel. Second Int. Congress of Radiation Research, Abstract, 60 (1962).

11 E.B. Ford. Ecological Genetics. Chapman and Hall, Great Britain (1971).

12 H.B.D. Kettlewell. Heredity 9, 323-342 (1955).

13 J. Magnusson and C. Ramel. Teratogenesis, Carcinogenesis and Mutagenesis 6, 289-305 (1986).

14 M.C. Singer, C.D. Thomas and C. Parmesan. Nature 366, 681-683 (1993).

$15 \mathrm{H}$. Croze. Zeitschrift für Tierpsychologie, Beiheft 5 (1970).

16 A.J. Cain, J.M. King and P.M. Sheppard. Genetics 45, 393-411 (1960).

17 A.J. Cain and P.M. Sheppard. Genetics 39, 89-116 (1954).

18 P.M. Sheppard. Natural Selection and Heredity. Hutchinson, London (1958).

19 C. Ramel. Hereditas 63, 15 (1969).

20 K. Ralls and R. Meadows. Nature 361, 689-690 (1993).

21 W.C. Orr and R.S. Sohal. Science 263, 1128-1130 (1994).

22 E.U. Selker. Trends in Genetics 13, 296-301 (1997).

23 J.A. Yoder, C.P. Walsh and T.H. Bestor. Trends in Genetics 13, 335-340 (1997).

24 G. Ising and C. Ramel. In The Genetics and Biology of Drosophila (M. Ashburner and E. Novitski, eds.), Vol. 1B, pp 947-954, Academic Press, New York (1976).

25 J.L. Marx. Science 224, 1415 (1984).

26 C. Biémond, A. Aouar and C. Arnault. Nature 329 (1987).

27 M.A. Houck, J.B. Clark, K.R. Peterson and M.G. Kidwell. Science 253, 1125-1129 (1991).

28 H.M. Robertson. Nature 362 (1993).

29 H.M. Robertson, K.L. Zumpano, A.R.Lohe, and D.L.Hartl. Nature Genetics 12, 360-361 (1996). 